\title{
On the Local Adjacency Metric Dimension of Generalized Petersen Graphs
}

\author{
Marsidi $^{1}$, Dafik$^{2}$, Ika Hesti Agustin ${ }^{3}$, Ridho Alfarisi ${ }^{4}$ \\ ${ }^{1}$ Mathematics Edu. Depart. IKIP PGRI Jember Indonesia \\ ${ }^{2}$ Mathematics Edu. Depart. University of Jember Indonesia \\ ${ }^{3}$ Mathematics Depart. University of Jember Indonesia \\ ${ }^{4}$ Elementary School Teacher Edu. Depart. University of Jember Indonesia
}

Email: marsidiarin@gmail.com,d.dafik@gmail.com,ikahesti@fmipa.unej.ac.id.

\begin{abstract}
The local adjacency metric dimension is one of graph topic. Suppose there are three neighboring vertex $a, b, c$ in path $a-c$. Path $a-c$ is called local if $a, b, c$ where each has representation: a is not equals $b$ and $a$ may equals to $c$. Let's say, $x, y \in V(G)$. For an order set of vertices $H=$ $\left\{h_{1}, h_{2}, \ldots, h_{k}\right\}$, the adjacency representation of $v$ with respect to $H$ is the ordered $k$-tuple $r_{A}\left(x_{j} \mid H\right)=\left(d_{A}\left(x, h_{1}\right), d_{A}\left(x, h_{2}\right), \ldots, d_{A}\left(x, h_{k}\right)\right)$, where $d_{A}(x, h)$ represents the adjacency distance $x-h$. The distance $d_{A}(x, h)$ defined by 0 if $x=h_{i}, 1$ if $x$ adjacent with $h$, and 2 if $x$ does not adjacent with $h$. The set $H$ is a local adjacency resolving set of $G$ if for every two distinct vertices $x, y$ and $x$ adjacent with y then $r_{A}\left(x_{j} \mid H\right)=r_{A}\left(y_{j} \mid H\right)$. A minimum local adjacency resolving set in $G$ is called local adjacency metric basis. The cardinality of vertices in the basis is a local adjacency metric dimension of $G$, denoted by $\left(\operatorname{dim}_{A, l}(G)\right)$. Next, we investigate the local adjacency metric dimension of generalized Petersen graph.
\end{abstract}

Keywords: Local Resolving Set; Local (Adjacency) Metric Dimension; Adjacency Metric Dimension; Generalized Petersen.

\section{INTRODUCTION}

A graph $G$ is defined by set of $V(G)$ and $E(G)$, the set of vertices and the set of edges of $G$, for more details of the definition in $[1,2]$. The metric dimension is one of interesting studied graph topics. Local means that every adjacent two vertices or two edges has distinct representation. Let's say, there are three neighboring vertex in a path, $a, b, c$ where each has representation: $a=b$ and $a$ may equals $c$. Then, the path $a-c$ is called local. The local adjacency metric dimension is combination of local metric dimension and adjacency metric dimension [3]. Let $G=(V, E)$ be a connected simple finite graph and $u$, $v$ in $G$. For an ordered set of vertices $X=\left\{x_{1}, x_{2}, \ldots, x_{k}\right\}$, the adjacency representation of $v$ with respect to $X$ is the ordered $k$-tuple $r_{A}(v \mid X)=\left(d_{A}\left(v, x_{1}\right), d_{A}\left(v, x_{2}\right), \ldots\right.$, $\left.d_{-} A\left(v, x_{-} k\right)\right)$, where $d_{A}(u, v)$ represents the adjacency distance $u-v \cdot d_{A}(u, v)$ defines by 0 if $u=v_{i}, 1$ if $u \sim v$, and 2 if $u \approx v$. We called $X$ is a local adjacency resolving set of $G$ if for every two distinct vertices $u, v$ and $u \sim v$ then $r_{A}(u \mid X)=r_{A}(v \mid X)$. A local adjacency metric basis of $\mathrm{G}$ is a minimum local adjacency resolving set in $G$. The cardinality of vertices in the basis is a local adjacency metric dimension of $G\left(\operatorname{dim}_{A, l}(G)\right)$.

The research is originated by Rodriguez, et al. [3] about local adjacency metric dimension of corona graphs. Marsidi, et. al. Determined the local metric dimension of line graph of special graphs. Then in 2017 Rinurwati, et al. [5] researched about local adjacency metric dimension of some wheel related graphs with pendant vertices. 
Recently, Darmaji, et al. [4] studied about local adjacency metric dimension of sun graph and stacked book graph this year.

\section{RESULTS AND DISCUSSION}

In this section, we investigate the local adjacency metric dimension of generalized Petersen graph $G P(n, k)$ for $k=2$ as follows

Theorem 1. The local adjacency metric dimension of $G P(n, 2)$ is $\operatorname{dim}_{A, l}(G P(n, 2))=$ $\frac{3 n+2}{7}$, for $n \equiv 4 \bmod 7$.

Proof. The vertex set of $G P(n, 2)$ is $V(G P(n, 2))=\left\{x_{i}, y_{i}: 1 \leq i \leq n\right\}$. We choose the local adjacensy resolving set $W=\left\{y_{i} ; i \equiv 1 \bmod 7\right\} \cup\left\{y_{n}-2\right\}$ such that we have the vertex representation respect to $H$ as follows.

$$
\begin{aligned}
& r\left(y_{i} \mid H\right)=\{\underbrace{2,2, \ldots,}_{\frac{3 n+2}{7}}\} \text {, for } i \equiv 3 \bmod 7 \\
& r\left(y_{i} \mid H\right)=\{\underbrace{2,2, \ldots, 2}_{\frac{3 n+2}{7}}\} \text {, for } i \equiv 6 \bmod 7 \\
& r\left(y_{n-1} \mid H\right)=\{\underbrace{2,2, \ldots, 2}_{\frac{3 n+2}{7}}\} \\
& r\left(y_{n} \mid H\right)=\{\underbrace{2,2, \ldots, 2}_{\frac{3 n+2}{7}}\} \\
& r\left(y_{i} \mid H\right)=\{\underbrace{2,2, \ldots,}_{\frac{i-2}{7}}, 1, \underbrace{2,2, \ldots, 2}_{\frac{3 n-i-3}{7}}\} \text {, for } i \equiv 2 \bmod 7 \\
& r\left(y_{i} \mid H\right)=\{\underbrace{2,2,2}_{\frac{i-3}{7}}, \ldots, \underbrace{2,2,2}_{\frac{i-2}{7}}, \underbrace{2, \ldots, 2}_{\frac{3 n-i-3}{7}}, 1, \underbrace{2, \ldots, 2}_{\frac{3 n-i-4}{7}}, \underbrace{2,2,2, \ldots, 2,2,2\}} \text {, for } i \equiv 4,5 \bmod 7 \\
& r\left(y_{i} \mid H\right)=\{\underbrace{2,2,2}_{\frac{i-3}{7}}, \ldots, \underbrace{2,2,2}_{\frac{i-2}{7}}, \underbrace{2, \ldots, 2}_{\frac{3 n-i-3}{7}}, 1, \underbrace{2, \ldots, 2}_{\frac{3 n-i-4}{7}}, \underbrace{2,2,2, \ldots, 2,2,2\}} \text {, for } i \equiv 0 \bmod 7
\end{aligned}
$$

The representation in vertex $x_{i} \in V(G P(n, 2))$ follows the representation in vertex $y_{i}$ such that we have the cardinality of local adjacency resolving set is $|H|=\mid\left\{v_{i} ; i \equiv\right.$ $1 \bmod 7\} \cup\left\{v_{n-2}\right\} \mid=\frac{3 n+2}{7}$. Thus, the upper bound of local adjacency metric dimension of $G P(n, 2)$ is $\operatorname{dim}_{A, l}(G P(n, 2)) \leq \frac{3 n+2}{7}$. Furthermore, we prove that the lower bound of local adjacency metric dimension of $G P(n, 2)$ is $\operatorname{dim}_{A, l}(G P(n, 2)) \geq \frac{3 n+2}{7}$. Assume that $\operatorname{dim}_{A, l}(G P(n, 2))<\frac{3 n+2}{7}$, we choose $|H|=\frac{3 n+2}{7}-1$ such that we have the same representation for two adjacent vertices in $G P(n, 2)$ namely $r\left(y_{n} \mid H\right) \neq r\left(y_{n-2} \mid H\right)=$. $\{\underbrace{2, \ldots,}_{\left(\frac{3 n-5}{7}\right)}$ Thus, the local adjacency metric dimension of $G P(n, 2)$ is $\operatorname{dim}_{A, l}(G P(n, 2))=\frac{3 n+2}{7}$, $\left(\frac{3 n-5}{7}\right)$

for $n \equiv 4 \bmod 7$.

Theorem 2. The local adjacency metric dimension of $G P(n, 2)$ is $\operatorname{dim}_{A, l}(G P(n, 2))=\frac{3 n+6}{7}$, for $n \equiv 5 \bmod 7$. 
Proof. The vertex set of $\operatorname{GP}(n, 2)$ is $\operatorname{V}(G P(n, 2))=\left\{x_{i}, y_{i}: 1 \leq i \leq n\right\}$. We choose the local adjacency resolving set $H=\left\{y_{i} ; i \equiv 1 \bmod 7\right\} \cup\left\{y_{n}-2\right\}$ such that we have the vertex representation respect to $H$ as follows.

$$
\begin{aligned}
& r\left(y_{i} \mid H\right)=\{\underbrace{2,2, \ldots,}_{\frac{3 n+2}{7}} 2\} \text {, for } i \equiv 3 \bmod 7 \\
& r\left(y_{i} \mid H\right)=\{\underbrace{2,2, \ldots,}_{\frac{3 n+2}{7}} 2\} \text {, for } i \equiv 6 \bmod 7 \\
& r\left(y_{n-1} \mid H\right)=\{\underbrace{2,2, \ldots, 2}_{\frac{3 n+2}{7}}\} \\
& r\left(y_{n} \mid H\right)=\{\underbrace{2,2, \ldots,}_{\frac{3 n+2}{7}} 2\} \\
& r\left(y_{i} \mid H\right)=\{\underbrace{2,2, \ldots,}_{\frac{i-2}{7}}, 2, \underbrace{1,2,2, \ldots}_{\frac{3 n-i-3}{7}}, 2\} \text {, for } i \equiv 2 \bmod 7 \\
& r\left(y_{i} \mid H\right)=\{\underbrace{2,2,2}_{\frac{i-3}{7}} \ldots, \underbrace{2,2,2}_{\frac{i-2}{7}}, \underbrace{2, \ldots, 2}_{\frac{3 n-i-3}{7}}, 1, \underbrace{2, \ldots, 2}_{\frac{3 n-i-4}{7}}, \underbrace{2,2,2, \ldots, 2,2,2\}} \text {, for } i \equiv 4,5 \bmod 7 \\
& r\left(y_{i} \mid H\right)=\{\underbrace{2,2,2}_{\frac{i-3}{7}}, \ldots, \underbrace{2,2,2}_{\frac{i-2}{7}}, \underbrace{2, \ldots, 2}_{\frac{3 n-i-3}{7}}, 1, \underbrace{2, \ldots, 2}_{\frac{3 n-i-4}{7}}, \underbrace{2,2,2, \ldots, 2,2,2\}}, \text { for } i \equiv 0 \bmod 7
\end{aligned}
$$

The representation in vertex $x_{i} \in V(G P(n, 2))$ follows the representation in vertex $y_{i}$ such that we have the cardinality of local adjacency resolving set is $|H|=\mid\left\{y_{i} ; i \equiv\right.$ $1 \bmod 7\} \cup\left\{y_{n-2}\right\} \mid=\frac{3 n+6}{7}$. Thus, the upper bound of local adjacency metric dimension of $G P(n, 2)$ is $\operatorname{dim}_{A, l}(G P(n, 2)) \leq \frac{3 n+6}{7}$. Furthermore, we prove that the lower bound of local adjacency metric dimension of $G P(n, 2)$ is $\operatorname{dim}_{A, l}(G P(n, 2)) \geq \frac{3 n+6}{7}$. Assume that $\operatorname{dim}_{A, l}(G P(n, 2))<\frac{3 n+6}{7}$, we choose $|H|=\frac{3 n+6}{7}-1$ such that we have the same representation for two adjacent vertices in $G P(n, 2)$ namely $r\left(y_{n} \mid H\right) \neq r\left(y_{n-2} \mid H\right)=$ $\{\underbrace{2, \ldots, 2\}}$. Thus, the local adjacency metric dimension of $G P(n, 2)$ is $\operatorname{dim}_{A, l}(G P(n, 2))=\frac{3 n+6}{7}$, $\left(\frac{3 n-5}{7}\right)$

for $n \equiv 5 \bmod 7$.

Theorem 3. The local adjacency metric dimension of $G P(n, 2)$ is $\operatorname{dim}_{A, l}(G P(n, 2))=\frac{3 n+3}{7}$, for $n \equiv 6 \bmod 7$.

Proof. The vertex set of $\operatorname{GP}(n, 2)$ is $V(G P(n, 2))=\left\{x_{i}, y_{i}: 1 \leq i \leq n\right\}$. We choose the local adjacency resolving set $H=\left\{y_{i} ; i \equiv 1 \bmod 7\right\} \cup\left\{y_{n}-2\right\}$ such that we have the vertex representation respect to $H$ as follows.

$$
\begin{aligned}
& r\left(y_{i} \mid H\right)=\{\underbrace{2,2, \ldots,}_{\frac{3 n+2}{7}}, 2\}, \text { for } i \equiv 3 \bmod 7 \\
& r\left(y_{i} \mid H\right)=\{\underbrace{2, \ldots, 2\}}, \text { for } i \equiv 6 \bmod 7 \\
& r\left(y_{n-1} \mid H\right)=\{2,2, \ldots, 2\}
\end{aligned}
$$




$$
\begin{aligned}
& \frac{3+2}{7} \\
& r\left(y_{n} \mid H\right)=\{\underbrace{2, \ldots, 2}_{\frac{3 n+2}{7}}\} \\
& r\left(y_{i} \mid H\right)=\{\underbrace{2,2, \ldots, 2}_{\frac{i-2}{7}}, 1, \underbrace{2,2, \ldots, 2}_{\frac{3 n-i-3}{7}}\} \text {, for } i \equiv 2 \bmod 7 \\
& r\left(y_{i} \mid H\right)=\{\underbrace{2,2,2}_{\frac{i-3}{7}}, \ldots, \underbrace{2,2,2}_{\frac{i-2}{7}}, \underbrace{2, \ldots, 2}_{\frac{3 n-i-3}{7}}, 1, \underbrace{2, \ldots, 2}_{\frac{3 n-i-4}{7}}, \underbrace{2,2, \ldots, 2,2,2\}} \text {, for } i \equiv 4,5 \bmod 7 \\
& r\left(y_{i} \mid H\right)=\{\underbrace{2,2,2, \ldots,}_{\frac{i-3}{7}}, \underbrace{2,2,2}_{\frac{i-2}{7}}, \underbrace{2, \ldots, 2}_{\frac{3 n-i-3}{7}}, 1, \underbrace{2, \ldots, 2}_{\frac{3 n-i-4}{7}}, \underbrace{2,2,2, \ldots, 2,2,2\}} \text {, for } i \equiv 0 \bmod 7
\end{aligned}
$$

The representation in vertex $x_{i} \in V(G P(n, 2))$ follows the representation in vertex $y_{i}$ such that we have the cardinality of local adjacency resolving set is $|H|=\mid\left\{y_{i} ; i \equiv\right.$ $1 \bmod 7\} \cup\left\{y_{n-2}\right\} \mid=\frac{3 n+3}{7}$. Thus, the upper bound of local adjacency metric dimension of $G P(n, 2)$ is $\operatorname{dim}_{A, l}(G P(n, 2)) \leq \frac{3 n+3}{7}$. Furthermore, we prove that the lower bound of local adjacency metric dimension of $G P(n, 2)$ is $\operatorname{dim}_{A, l}(G P(n, 2)) \geq \frac{3 n+3}{7}$. Assume that $\operatorname{dim}_{A, l}(G P(n, 2))<\frac{3 n+3}{7}$, we choose $|H|=\frac{3 n+3}{7}-1$ such that we have the same representation for two adjacent vertices in $G P(n, 2)$ namely $r\left(y_{n} \mid H\right) \neq r\left(y_{n-2} \mid H\right)=$ $\{\underbrace{2, \ldots, 2\}}_{\left(\frac{3 n-5}{7}\right)}$. Thus, the local adjacency metric dimension of $G P(n, 2)$ is $\operatorname{dim}_{A, l}(G P(n, 2))=\frac{3 n+3}{7}$, for $n \equiv 6 \bmod 7$.

Theorem 4. The local adjacency metric dimension of $G P(n, 2)$ is $\operatorname{dim}_{A, l}(G P(n, 2))=\frac{3 n}{7}$, for $n \equiv 0 \bmod 7$.

Proof. The vertex set of $G P(n, 2)$ is $V(G P(n, 2))=\left\{x_{i}, y_{i}: 1 \leq i \leq n\right\}$. We choose the local adjacency resolving set $H=\left\{y_{i} ; i \equiv 1 \bmod 7\right\} \cup\left\{y_{n}-2\right\}$ such that we have the vertex representation respect to $H$ as follows.

$$
\begin{aligned}
& r\left(y_{i} \mid H\right)=\{\underbrace{2,2, \ldots, 2}_{\frac{3 n+2}{7}}\} \text {, for } i \equiv 3 \bmod 7 \\
& r\left(y_{i} \mid H\right)=\{\underbrace{2,2, \ldots, 2}_{\frac{3 n+2}{7}}\} \text {, for } i \equiv 6 \bmod 7 \\
& r\left(y_{n-1} \mid H\right)=\{\underbrace{2,2, \ldots, 2}_{\frac{3 n+2}{7}}\} \\
& r\left(y_{n} \mid H\right)=\{\underbrace{2,2, \ldots,}_{\frac{3 n+2}{7}} 2\} \\
& r\left(y_{i} \mid H\right)=\{\underbrace{2,2, \ldots, 2}_{\frac{i-2}{7}}, 1, \underbrace{2,2, \ldots,}_{\frac{3 n-i-3}{7}}, 2\} \text {, for } i \equiv 2 \bmod 7 \\
& r\left(y_{i} \mid H\right)=\{2,2,2, \ldots, \underbrace{2,2,2}, \underbrace{2, \ldots, 2}, 1, \underbrace{2, \ldots, 2}, \underbrace{2,2,2}, \ldots, 2,2,2\}, \text { for } i \equiv 4,5 \bmod 7
\end{aligned}
$$




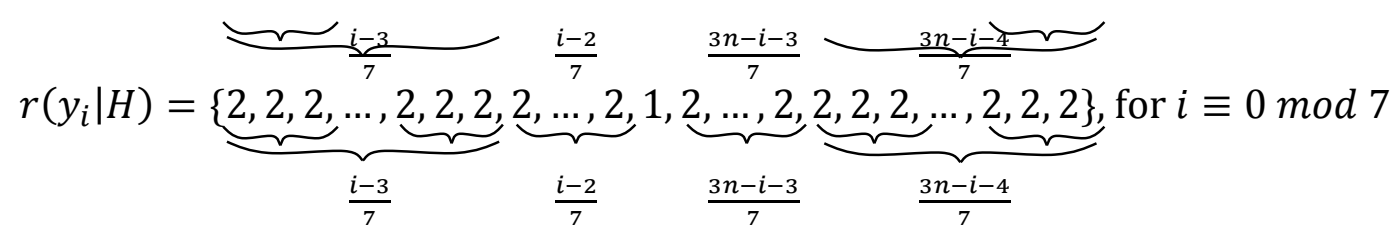

The representation in vertex $x_{i} \in V(G P(n, 2))$ follows the representation in vertex $y_{i}$ such that we have the cardinality of local adjacency resolving set is $|H|=\mid\left\{y_{i} ; i \equiv\right.$ $1 \bmod 7\} \cup\left\{y_{n-2}\right\} \mid=\frac{3 n+2}{7}$. Thus, the upper bound of local adjacency metric dimension of $G P(n, 2)$ is $\operatorname{dim}_{A, l}(G P(n, 2)) \leq \frac{3 n}{7}$. Furthermore, we prove that the lower bound of local adjacency metric dimension of $G P(n, 2)$ is $\operatorname{dim}_{A, l}(G P(n, 2)) \geq \frac{3 n}{7}$. Assume that $\operatorname{dim}_{A, l}(G P(n, 2))<\frac{3 n}{7}$, we choose $|H|=\frac{3 n}{7}-1$ such that we have the same representation for two adjacent vertices in $G P(n, 2)$ namely $r\left(y_{n} \mid H\right) \neq r\left(y_{n-2} \mid H\right)=\{\underbrace{2, \ldots, 2\}}$. Thus, the $\underbrace{2,0}_{\left(\frac{3 n-5}{7}\right)}$

local adjacency metric dimension of $G P(n, 2)$ is $\operatorname{dim}_{A, l}(G P(n, 2))=\frac{3 n}{7}$, for $n \equiv 0 \bmod 7$.

Theorem 5. The local adjacency metric dimension of $G P(n, 2)$ is $\operatorname{dim}_{A, l}(G P(n, 2))=\frac{3 n+4}{7}$, for $n \equiv 1 \bmod 7$.

Proof. The vertex set of $\operatorname{GP}(n, 2)$ is $\operatorname{V}(G P(n, 2))=\left\{x_{i}, y_{i}: 1 \leq i \leq n\right\}$. We choose the local adjacency resolving set $H=\left\{y_{i} ; i \equiv 1 \bmod 7\right\} \cup\left\{y_{n}-2\right\}$ such that we have the vertex representation respect to $H$ as follows.

$$
\begin{aligned}
& r\left(y_{i} \mid H\right)=\{\underbrace{2,2, \ldots, 2}_{\frac{3 n+2}{7}}\} \text {, for } i \equiv 3 \bmod 7 \\
& r\left(y_{i} \mid H\right)=\{\underbrace{2,2, \ldots, 2}_{\frac{3 n+2}{7}}\} \text {, for } i \equiv 6 \bmod 7 \\
& r\left(y_{n-1} \mid H\right)=\{\underbrace{2,2, \ldots, 2}_{\frac{3 n+2}{7}}\} \\
& r\left(y_{n} \mid H\right)=\{\underbrace{2, \ldots, 2}_{\frac{3 n+2}{7}}\} \\
& r\left(y_{i} \mid H\right)=\{\underbrace{2,2, \ldots, 2}_{\frac{i-2}{7}}, 1, \underbrace{2,2, \ldots, 2}_{\frac{3 n-i-3}{7}}\} \text {, for } i \equiv 2 \bmod 7 \\
& r\left(y_{i} \mid H\right)=\{\underbrace{2,2,2}_{\frac{i-3}{7}}, \ldots, \underbrace{2,2,2}_{\frac{i-2}{7}}, \underbrace{2, \ldots, 2}_{\frac{3 n-i-3}{7}}, 1, \underbrace{2, \ldots, 2}_{\frac{3 n-i-4}{7}}, \underbrace{2,2,2, \ldots, 2,2\}}, \text { for } i \equiv 4,5 \bmod 7 \\
& r\left(y_{i} \mid H\right)=\underbrace{22,2,2}_{\frac{i-3}{7}}, \ldots, \underbrace{2,2,2}_{\frac{i-2}{7}}, \underbrace{2, \ldots, 2}_{\frac{3 n-i-3}{7}}, 1, \underbrace{2, \ldots, 2}_{\frac{3 n-i-4}{7}}, \underbrace{2,2,2} \ldots, \underbrace{2,2,2\}}, \text { for } i \equiv 0 \bmod 7
\end{aligned}
$$

The representation in vertex $x_{i} \in V(G P(n, 2))$ follows the representation in vertex $y_{i}$ such that we have the cardinality of local adjacency resolving set is $|H|=\mid\left\{y_{i} ; i \equiv\right.$ $1 \bmod 7\} \cup\left\{y_{n-2}\right\} \mid=\frac{3 n+4}{7}$. Thus, the upper bound of local adjacency metric dimension of $G P(n, 2)$ is $\operatorname{dim}_{A, l}(G P(n, 2)) \leq \frac{3 n+4}{7}$. Furthermore, we prove that the lower bound of local 
adjacency metric dimension of $G P(n, 2)$ is $\operatorname{dim}_{A, l}(G P(n, 2)) \geq \frac{3 n+4}{7}$. Assume that $\operatorname{dim}_{A, l}(G P(n, 2))<\frac{3 n+4}{7}$, we choose $|H|=\frac{3 n}{7}-1$ such that we have the same representation for two adjacent vertices in $G P(n, 2)$ namely $r\left(y_{n} \mid H\right) \neq r\left(y_{n-2} \mid H\right)=$ $\{\underbrace{2, \ldots, 2\}}$. Thus, the local adjacency metric dimension of $\operatorname{GP}(n, 2)$ is $\operatorname{dim}_{A, l}(G P(n, 2))=\frac{3 n+4}{7}$, $\left(\frac{3 n-5}{7}\right)$

for $n \equiv 1 \bmod 7$.

Theorem 6. The local adjacency metric dimension of $G P(n, 2)$ is $\operatorname{dim}_{A, l}(G P(n, 2))=\frac{3 n+1}{7}$, for $n \equiv 2 \bmod 7$.

Proof. The vertex set of $G P(n, 2)$ is $V(G P(n, 2))=\left\{x_{i}, y_{i}: 1 \leq i \leq n\right\}$. We choose the local adjacency resolving set $H=\left\{y_{i} ; i \equiv 1 \bmod 7\right\} \cup\left\{y_{n}-2\right\}$ such that we have the vertex representation respect to $H$ as follows.

$$
\begin{aligned}
& r\left(y_{i} \mid H\right)=\{\underbrace{2,2, \ldots,}_{\frac{3 n+2}{7}}\} \text {, for } i \equiv 3 \bmod 7 \\
& r\left(y_{i} \mid H\right)=\{\underbrace{2,2, \ldots, 2}_{\frac{3 n+2}{7}}\} \text {, for } i \equiv 6 \bmod 7 \\
& r\left(y_{n-1} \mid H\right)=\{\underbrace{2,2, \ldots, 2}_{\frac{3 n+2}{7}}\} \\
& r\left(y_{n} \mid H\right)=\{\underbrace{2,2, \ldots,}_{\frac{3 n+2}{7}}, 2\} \\
& r\left(y_{i} \mid H\right)=\{\underbrace{2,2, \ldots, 2}_{\frac{i-2}{7}}, 1, \underbrace{2,2, \ldots,}_{\frac{3 n-i-3}{7}}\} \text {, for } i \equiv 2 \bmod 7 \\
& r\left(y_{i} \mid H\right)=\{\underbrace{2,2,2}_{\frac{i-3}{7}} \ldots, \underbrace{2,2,2}_{\frac{i-2}{7}}, \underbrace{2, \ldots, 2}_{\frac{3 n-i-3}{7}}, 1, \underbrace{2, \ldots, 2}_{\frac{3 n-i-4}{7}}, \underbrace{2,2, \ldots,}, \underbrace{2,2,2\}} \text {, for } i \equiv 4,5 \bmod 7 \\
& r\left(y_{i} \mid H\right)=\{\underbrace{2,2,2}_{\frac{i-3}{7}}, \ldots, \underbrace{2,2,2}_{\frac{i-2}{7}}, \underbrace{2, \ldots, 2}_{\frac{3 n-i-3}{7}}, 1, \underbrace{2, \ldots, 2}_{\frac{3 n-i-4}{7}}, \underbrace{2,2,2, \ldots, \underbrace{2,2,2\}}} \text {, for } i \equiv 0 \bmod 7
\end{aligned}
$$

The representation in vertex $x_{i} \in V(G P(n, 2))$ follows the representation in vertex $y_{i}$ such that we have the cardinality of local adjacency resolving set is $|H|=\mid\left\{y_{i} ; i \equiv\right.$ $1 \bmod 7\} \cup\left\{y_{n-2}\right\} \mid=\frac{3 n+1}{7}$. Thus, the upper bound of local adjacency metric dimension of $G P(n, 2)$ is $\operatorname{dim}_{A, l}(G P(n, 2)) \leq \frac{3 n+1}{7}$. Furthermore, we prove that the lower bound of local adjacency metric dimension of $G P(n, 2)$ is $\operatorname{dim}_{A, l}(G P(n, 2)) \geq \frac{3 n+1}{7}$. Assume that $\operatorname{dim}_{A, l}(G P(n, 2))<\frac{3 n+1}{7}$, we choose $|H|=\frac{3 n+1}{7}-1$ such that we have the same representation for two adjacent vertices in $G P(n, 2)$ namely $r\left(y_{n} \mid H\right) \neq r\left(y_{n-2} \mid H\right)=$ $\{\underbrace{}_{\left(\frac{3 n}{7}, \ldots, 2\right\}}$. Thus, the local adjacency metric dimension of $\operatorname{GP}(n, 2)$ is $\operatorname{dim}_{A, l}(G P(n, 2))=\frac{3 n+1}{7}$, $\underbrace{2, n}_{\left(\frac{3 n-5}{7}\right)}$

for $n \equiv 2 \bmod 7$. 
Theorem 7. The local adjacency metric dimension of $G P(n, 2)$ is $\operatorname{dim}_{A, l}(G P(n, 2))=\frac{3 n-2}{7}$, for $n \equiv 3 \bmod 7$.

Proof. The vertex set of $\operatorname{GP}(n, 2)$ is $\operatorname{V(GP}(n, 2))=\left\{x_{i}, y_{i}: 1 \leq i \leq n\right\}$. We choose the local adjacency resolving set $H=\left\{y_{i} ; i \equiv 1 \bmod 7\right\} \cup\left\{y_{n}-2\right\}$ such that we have the vertex representation respect to $H$ as follows.

$$
\begin{aligned}
& r\left(y_{i} \mid H\right)=\{\underbrace{2,2, \ldots, 2}_{\frac{3 n+2}{7}}\} \text {, for } i \equiv 3 \bmod 7 \\
& r\left(v_{i} \mid H\right)=\{\underbrace{2,2, \ldots, 2}_{\frac{3 n+2}{7}}\} \text {, for } i \equiv 6 \bmod 7 \\
& r\left(y_{n-1} \mid H\right)=\{\underbrace{2,2, \ldots, 2}_{\frac{3 n+2}{7}}\} \\
& r\left(y_{n} \mid H\right)=\{\underbrace{2,2, \ldots,}_{\frac{3 n+2}{7}}, 2\} \\
& r\left(y_{i} \mid H\right)=\{\underbrace{2,2, \ldots,}_{\frac{i-2}{7}}, 1, \underbrace{2,2, \ldots, 2}_{\frac{3 n-i-3}{7}}\} \text {, for } i \equiv 2 \bmod 7 \\
& r\left(y_{i} \mid H\right)=\{\underbrace{2,2,2}_{\frac{i-3}{7}} \ldots, \underbrace{2,2,2}_{\frac{i-2}{7}}, \underbrace{2, \ldots, 2}_{\frac{3 n-i-3}{7}}, 1, \underbrace{2, \ldots, 2}_{\frac{3 n-i-4}{7}}, \underbrace{2,2,2, \ldots, 2,2\}}, \text { for } i \equiv 4,5 \bmod 7 \\
& r\left(y_{i} \mid H\right)=\{\underbrace{2,2,2}_{\frac{i-3}{7}}, \ldots, \underbrace{2,2,2}_{\frac{i-2}{7}}, \underbrace{2, \ldots, 2}_{\frac{3 n-i-3}{7}}, 1, \underbrace{2, \ldots, 2}_{\frac{3 n-i-4}{7}}, \underbrace{2,2,2, \ldots, 2,2,2\}} \text {, for } i \equiv 0 \bmod 7
\end{aligned}
$$

The representation in vertex $x_{i} \in V(G P(n, 2))$ follows the representation in vertex $y_{i}$ such that we have the cardinality of local adjacency resolving set is $|H|=\mid\left\{y_{i} ; i \equiv\right.$ $1 \bmod 7\} \cup\left\{y_{n-2}\right\} \mid=\frac{3 n-2}{7}$. Thus, the upper bound of local adjacency metric dimension of $G P(n, 2)$ is $\operatorname{dim}_{A, l}(G P(n, 2)) \leq \frac{3 n-2}{7}$. Furthermore, we prove that the lower bound of local adjacency metric dimension of $G P(n, 2)$ is $\operatorname{dim}_{A, l}(G P(n, 2)) \geq \frac{3 n-2}{7}$. Assume that $\operatorname{dim}_{A, l}(G P(n, 2))<\frac{3 n-2}{7}$, we choose $|H|=\frac{3 n-2}{7}-1$ such that we have the same representation for two adjacent vertices in $G P(n, 2)$ namely $r\left(y_{n} \mid H\right) \neq r\left(y_{n-2} \mid H\right)=$ $\{\underbrace{2, \ldots, 2\}}$. Thus, the local adjacency metric dimension of $\operatorname{GP}(n, 2)$ is $\operatorname{dim}_{A, l}(G P(n, 2))=\frac{3 n-2}{7}$, $\underbrace{}_{\left(\frac{3 n-5}{7}\right)}$

for $n \equiv 3 \bmod 7$.

\section{CONCLUSIONS}

We have discussed about the local adjacency metric dimension of generalized Petersen graph $G P(n, k)$ for $k=2$. Accordingly, we have some problem for $k \geq 3$ as follows.

Open Problem 1. Find the local adjacency metric dimension of generalized Petersen graph $G P(n, k)$ for $k \geq 3$ ?. 


\section{ACKNOWLEDGMENTS}

We gratefully acknowledge the support from IKIP PGRI Jember 2019 and CGANTUniversity of Jember of year 2019.

\section{REFERENCES}

[1] J. L. Gross, J. Yellen and P. Zhang, Handbook of graph Theory, Second Edition CRC Press Taylor and Francis Group, 2014.

[2] G. Chartrand and L. Lesniak, Graphs and digraphs 3rd ed London: Chapman and Hall, 2000.

[3] J. A. Rodriguez-Velazquez and H. Fernau, "On the (adjacency) metric dimension of corona and strong product graphs and their local variants", Combinatorial and Computational Results, Arxiv: 1309.2275.v1[math.CO].

[4] A. Y. Badri and Darmaji, "Local adjacency metric dimension of sun graph and stacked book graph", Journal of Physics: Conf. Series, vol. 974, No. 012069, pp. 01-05, 2018.

[5] Rinurwati, H. Suprajitno, and Slamin, "On local adjacency metric dimension of some wheel related graphs with pendant points", AIP Conference Proceedings, vol. 1867, No. 020065, pp. 01-06, 2017.

[6] Marsidi, M., Dafik, D., Agustin, I. H., \& Alfarisi, R. (2016). On the local metric dimension of line graph of special graph. Cauchy, 4(3), 125-130. 